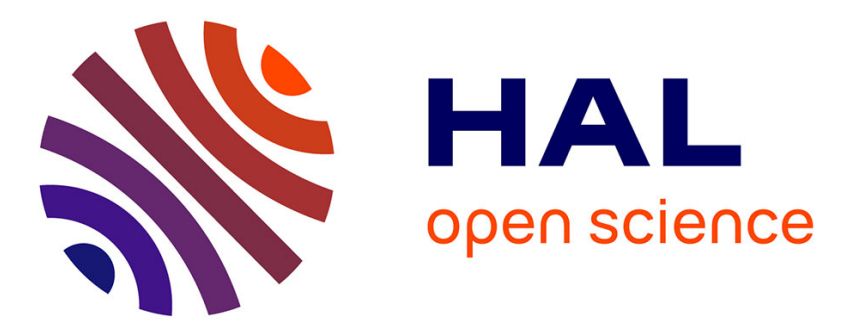

\title{
Interpretation of Experimental Soret Bands of Porphyrins in Flexible Covalent Cages and in Their Related Ag(I) Fixed Complexes
}

Laura Zanetti-Polzi, Andrea Amadei, Ryan Djemili, Stephanie Durot, Laëtitia Schoepff, Valérie Heitz, Barbara Ventura, Isabella Daidone

\section{To cite this version:}

Laura Zanetti-Polzi, Andrea Amadei, Ryan Djemili, Stephanie Durot, Laëtitia Schoepff, et al.. Interpretation of Experimental Soret Bands of Porphyrins in Flexible Covalent Cages and in Their Related Ag(I) Fixed Complexes. Journal of Physical Chemistry C, 2019, 123 (20), pp.13094-13103. 10.1021/acs.jpcc.9b00742 . hal-03415517

\section{HAL Id: hal-03415517 https://hal.science/hal-03415517}

Submitted on 4 Nov 2021

HAL is a multi-disciplinary open access archive for the deposit and dissemination of scientific research documents, whether they are published or not. The documents may come from teaching and research institutions in France or abroad, or from public or private research centers.
L'archive ouverte pluridisciplinaire HAL, est destinée au dépôt et à la diffusion de documents scientifiques de niveau recherche, publiés ou non, émanant des établissements d'enseignement et de recherche français ou étrangers, des laboratoires publics ou privés. 


\title{
- Interpretation of Experimental Soret Bands of Porphyrins in Flexible ${ }_{2}$ Covalent Cages and in Their Related Ag(I) Fixed Complexes
}

\author{
3 Laura Zanetti-Polzi, ${ }^{\dagger}$ A Andrea Amadei, ${ }^{\dagger}$ Ryan Djemili, ${ }^{\S}$ Stéphanie Durot, ${ }^{\S}$ Laetitia Schoepff, ${ }^{\S}$

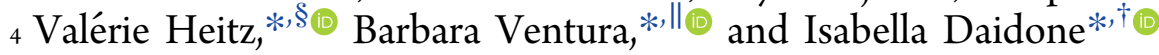 \\ $5{ }^{\dagger}$ Department of Physical and Chemical Sciences, University of L'Aquila, via Vetoio (Coppito 1), 67010 L'Aquila, Italy \\ $6{ }^{\ddagger}$ Department of Chemical and Technological Sciences, University of Rome "Tor Vergata”, Via della Ricerca Scientifica, 00185 Rome, \\ 7 Italy \\ $8{ }^{8}$ Laboratoire de Synthèse des Assemblages Moléculaires Multifonctionnels, Institut de Chimie de Strasbourg, CNRS/UMR 7177, \\ 9 Université de Strasbourg, 67008 Strasbourg Cedex, France \\ 10 "Institute for Organic Synthesis and Photoreactivity (ISOF)-National Research Council (CNR), Via P. Gobetti 101, 40129 \\ 11 Bologna, Italy
}

12 S Supporting Information

13 ABSTRACT: The essential features of the experimental 14 Soret bands of two covalent cages, consisting of two zinc15 porphyrins connected by four flexible spacers, are for the first 16 time interpreted and characterized at a molecular level by 17 means of a mixed quantum/classical procedure based on 18 molecular dynamics (MD) simulation and the perturbed 19 matrix method (PMM). The same method allows also for a 20 comprehensive interpretation of the changes in the UV21 visible absorbance of the cages upon silver(I) complexation to 22 the peripheral binding sites. Although the zinc-to-zinc 23 distance is found to be similar in both cages, the MD-PMM

24 calculations show that the conformation adopted by the cage with longer linkers corresponds to more slipped porphyrins, giving 25 rise to a red-shifted $(7-8 \mathrm{~nm})$, broader, and slightly split Soret peak with respect to the cage with shorter linkers. The process of 26 silver(I) complexation separates the two porphyrins in a face-to-face conformation in both cages, resulting in narrower (and 27 more similar) Soret bands due to a reduced excitonic coupling. Despite the similar features of the spectra of the two silver(I)28 complexed cages, a slight difference in the peak maxima of about $2 \mathrm{~nm}$ is observed, arising from a slightly shorter zinc-to-zinc 29 distance in the cage with longer linkers. These results show that the MD-PMM methodology is a reliable method to obtain 30 information on the relative disposition and exciton coupling interaction of porphyrins in flexible systems in solution, from the 31 analysis of their absorption spectra.
33 The first steps in natural photosynthesis rely on the collective 34 work of chlorophyll or bacteriochlorophyll organized in 35 antennas to capture light and on their precise arrangement 36 within the reaction centers to convert light energy into 37 chemical energy. ${ }^{1-7}$ The distance and mutual orientation of 38 these chromophores are critical as they determine their 39 absorption range and their efficiency as energy donors or 40 acceptors or as redox active species. ${ }^{8}$ Therefore, engineering 41 systems incorporating porphyrins, tetrapyrrolic macrocycles 42 related to these natural chromophores, has led to various light 43 converting systems. ${ }^{9-20}$ Molecular cages incorporating two or 44 more porphyrins have shown their ability to respond to light 45 and also to work as nanoreceptors or nanoreactors, depending 46 on the specific arrangement and orientation of the porphyr47 ins. ${ }^{11,21-26}$ Related to these various applications and as a tool 48 to rationalize and design systems with specific functions, 49 optical spectra of porphyrin architectures can give important information on the electronic interactions among the single 50 units, which depend on the geometry of the system. Indeed, 51 spectral features of the Soret (or B) band of assembled close- 52 lying porphyrins with strong electronic coupling are largely 53 affected by the relative position of the monomers. The position 54 and splitting of the Soret band, which are defined by the 55 relative orientation of the two $B$ transitions of each 56 chromophore, $\mathrm{B}_{x}$ and $\mathrm{B}_{y}$, are commonly qualitatively 57 interpreted in the frame of the transition dipole exciton 58 coupling theory. ${ }^{11,13,26-31}$ Nevertheless, a quantitative recon- 59 struction, and interpretation, of the experimental absorption 60 spectra of flexible assemblies in solution, such as covalent cages 61 consisting of two porphyrins connected by four flexible 62 spacers, is challenging.

Received: January 24, 2019

Revised: April 16, 2019

Published: April 29, 2019 
64 Here, a combined spectroscopic and computational 65 approach is presented to study different flexible porphyrinic 66 cages. Cages $\mathbf{1}$ and $\mathbf{2}$ and their corresponding silver(I)67 complexed cages, $\left[\mathrm{Ag}_{4}(1)\right]^{4+}$ and $\left[\operatorname{Ag}_{4}(2)\right]^{4+}$, discussed in the
Scheme 1. Chemical Structure of the cages 2 d 2, of Their Silver(I) Complexed Forms (with the Coordination Sphere of $\operatorname{Ag}(\mathrm{I})$ Represented as Discussed Below) and of the Reference Compound, Zn-Alkyne
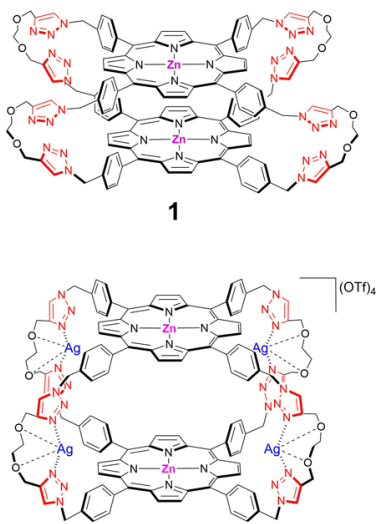

$\left[\operatorname{Ag}_{4}(\mathbf{1})\right] \mathrm{OTf} 4$
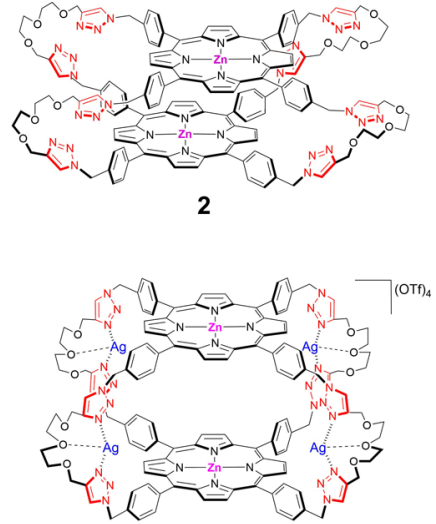

$\left[\mathrm{Ag}_{4}(2)\right] \mathrm{OTf}_{4}$

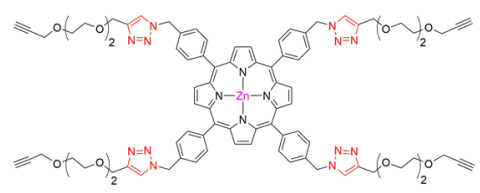

Zn-alkyne

69 are covalent cages consisting of two zinc(II) tetraphenylpor70 phyrins, Zn-TPPs, connected by four flexible connectors of 71 different lengths, each incorporating two 1,2,3-triazolyl ligands. 72 The linkers of cage $\mathbf{1}$ that incorporate one ethyleneglycol unit 73 are shorter than the ones of cage $\mathbf{2}$ that incorporate a 74 diethyleneglycol unit. Binding of four silver(I) ions to the 75 peripheral ligands induces large conformational changes in 76 solution and rigidizes the cages. These conformational changes 77 are reversible: removal of $\mathrm{Ag}(\mathrm{I})$ in the presence of an excess 01 78 chloride anions switches back both cages to their collap79 conformations. ${ }^{32,33}$ The strength of the multimolecular binding 80 process with silver(I) ions is here determined with UV-visible 81 absorption titrations for cages $\mathbf{1}$ and 2 . Absorption and 82 emission properties of the two cages and of their complexes $83\left[\operatorname{Ag}_{4}(1)\right]^{4+}$ and $\left[\operatorname{Ag}_{4}(2)\right]^{4+}$ are characterized in solution by 84 means of steady-state and time-resolved spectroscopic 85 techniques.

86 The computational strategy used in the present work to 87 calculate the UV-visible absorption spectra in the Soret region 88 of the experimentally studied cages is based on the perturbed 89 matrix method (PMM) $)^{34,35}$ and molecular dynamics (MD) 90 simulations. With the MD-PMM approach, the Soret band of 91 highly flexible, close-lying porphyrinic assemblies in solution 92 can be reconstructed, allowing a quantitative interpretation of 93 the experimental spectra at an atomic detail. The main 94 strengths of this computational approach are that: (i) a very 95 large number of conformations (including the whole system 96 and the solvent atoms) can be analyzed because the procedure 97 makes use of classical MD simulation for the conformational sampling; (ii) the effects on the band shape and position 98 arising from the electrostatic perturbation of the environment 99 (i.e., the solvent and the rest of the system, excluding the 100 chromophore) and the excitonic coupling (treated as dipole- 101 dipole interaction) can be separated and their contributions 102 analyzed. The MD-PMM methodology is here applied for the 103 first time to porphyrinic assemblies, the modeling of which is a 104 topic of current and broad interest. ${ }^{36}$ The very good agreement 105 between experimental and theoretical results gives high 106 credence to this method to get geometrical data of interacting 107 porphyrins in complex architectures, simply from their 108 absorption spectra.

109

\section{RESULTS AND DISCUSSION}

110

Experimental Results. Absorption and Emission Proper- 111 ties of Cages 1 and 2. The absorption and the emission 112 features of the two cages are characterized in dichloromethane 113 $(\mathrm{DCM}) / \mathrm{MeOH}(90: 10)$ and compared with those of $\mathrm{Zn}-114$ alkyne (Scheme 1), ${ }^{32}$ which is considered as a monomeric 115 model for the cages. The absorption spectra of $\mathbf{1}$ and $\mathbf{2}$ are 116 shown in Figure 1, together with twice the absorption $117 \mathrm{fl}$

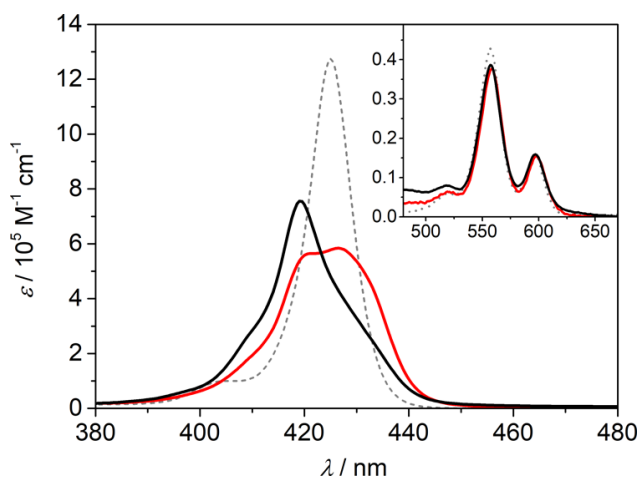

Figure 1. Absorption spectra of cage 1 (black), cage 2 (red), and twice the absorption spectrum of Zn-alkyne (gray, dashed) in DCM/ $\mathrm{MeOH}$ (90:10) in the Soret region $\left(\mathrm{S}_{0} \rightarrow \mathrm{S}_{2}\right.$ transition). In the inset, the $480-670 \mathrm{~nm}$ region (Q-band region) is shown.

spectrum of Zn-alkyne (the absorption data are collected in Table 1). The Soret bands in the cages are broadened $119 \mathrm{tl}$

Table 1. Absorption Parameters in DCM/MeOH (90:10)

$\begin{array}{ccc} & \lambda_{\max } / \mathrm{nm} & \varepsilon / 10^{5} \mathrm{M}^{-1} \mathrm{~cm}^{-1} \\ \text { Zn-alkyne } & 425 & 6.37 \\ & 557 & 0.21 \\ & 597 & 0.08 \\ & 419 & 7.55 \\ & 558 & 0.39 \\ & 597 & 0.16 \\ & 421 & 5.65 \\ & 426 & 5.83 \\ & 558 & 0.38 \\ 597 & 0.15\end{array}$

compared to the sum of two model porphyrins, and their 120 features are different in the two cases, showing a splitting in 121 cage 2. The Q-band region (inset of Figure 1), conversely, is 122 nearly identical for 1, 2, and the sum of two Zn-alkyne units. 123 The integrated molar absorption coefficients, calculated on the 124 whole absorption spectrum, are $1.33 \times 10^{9}$ and $1.32 \times 10^{9} \mathrm{M}^{-1} 125$ 
$126 \mathrm{~cm}^{-2}$ for cages 1 and 2 , respectively, and equal to twice the 127 integrated absorption coefficient of Zn-alkyne $\left(6.62 \times 10^{8} \mathrm{M}^{-1}\right.$ $\left.128 \mathrm{~cm}^{-2}\right)$. This is indicative of exciton coupling within the pair of 129 identical chromophores in the cages, leading to the observed 130 spectral features. It can be noticed that although both the Soret 131 and $Q$ bands arise from transitions from the same molecular 132 orbitals, the very different magnitudes of the corresponding 133 transition moments make the $\mathrm{Q}$ absorption bands insensitive 134 to the exciton coupling. A detailed computational analysis of 135 the origin of the observed spectra is presented in the following 136 section.

137 Fluorescence spectra, recorded both at room temperature 138 and at $77 \mathrm{~K}$ in $\mathrm{DCM} / \mathrm{MeOH}$ (50:50), are very similar for the 139 cages and the model, with maxima at ca. 606 and $660 \mathrm{~nm}$ at 140 room temperature and 600 and $660 \mathrm{~nm}$ at low temperature, 141 respectively, with a slight red-shift for the cages in the latter 142 case (Figures S1 and S2 and Table S1). Moreover, the 143 emission quantum yields and the singlet excited state lifetimes 144 of the cages are almost identical to those of Zn-alkyne $(0.040$ 145 and $1.7 \mathrm{~ns}$, respectively, Table S1). By means of gated 146 detection, the phosphorescence spectra of the porphyrins have 147 been isolated at $77 \mathrm{~K}$, revealing a small red shift $(6-7 \mathrm{~nm})$ in 148 the phosphorescence maxima of the cages compared to the 149 model (Figure S2 inset and Table S1). The triplet excited state 150 lifetimes are in the order of $20 \mathrm{~ms}$ in all cases. Overall, the 151 luminescence data indicate that the emission pathways of the 152 porphyrins are almost unaffected by the con trained environ153 ment of the cage, contrary to what w... bserved for the 154 absorption features which are strongly dependent on the 155 conformation of the systems.

$156 \mathrm{Ag}^{+}$Complexation of Cages 1 and 2 in Solution. In order 157 to ch 2 erize the process of complexation of the peripheral 158 triazolyl ligands with silver(I) ions, cages 1 and 2 have bee 159 titrated with $\mathrm{Ag}(\mathrm{OTf})$ in $\mathrm{DCM} / \mathrm{MeOH}$ (90:10), afte 160 absorption and emission changes by means of spectrophoto161 metric and spectrofluorimetric analysis. Addition of increasing 162 amounts of $\mathrm{Ag}^{+}$ions to a solution of $2\left(6.1 \times 10^{-7} \mathrm{M}\right)$ causes 163 significant modifications in the absorption spectrum of the 164 cage, as shown in Figure 2. In the Soret region, the splitting is 165 progressively reduced with depression of the absorption in the $166430-440 \mathrm{~nm}$ region and increase of a single band at $419.5 \mathrm{~nm}$. 167 A nearly biphasic behavior is observed, with the isosbestic 168 point shifting from 425 to $424 \mathrm{~nm}$. The Q-bands are only 169 slightly affected by the addition of $\mathrm{Ag}(\mathrm{I})$ (Figure 2 inset).

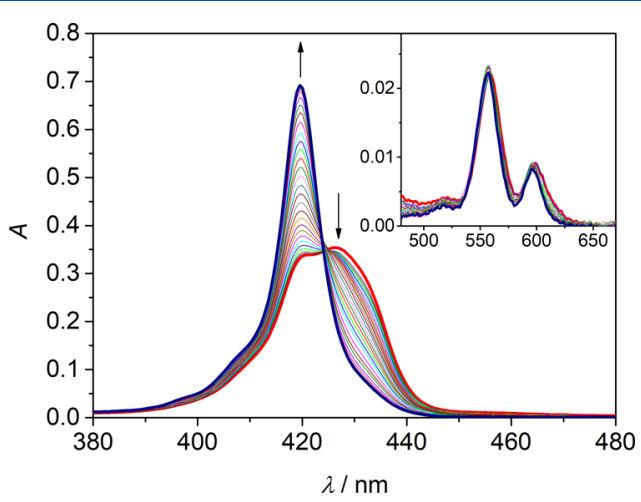

Figure 2. Absorption spectra of a solution of $2\left(6.1 \times 10^{-7} \mathrm{M}\right)$ in $\mathrm{DCM} / \mathrm{MeOH}(90: 10)$ upon addition of $\mathrm{Ag}(\mathrm{OTf}):\left[\mathrm{Ag}^{+}\right]=0-16.5$ equiv (red thick: 0 equiv, blue thick: 16.5 equiv; the arrows indicate the trend of the curves). In the inset, the Q-band region is shown.
Emission spectra, collected upon excitation both at $424 \mathrm{~nm} 170$ (isosbestic point of the Soret region, Figure S3) and at $558 \mathrm{~nm} 171$ (invariant point of the Q-band region, Figure S4), show a clear 172 biphasic trend but with an overall small modification of the 173 emission features: in the range $0-10$ equiv, a slight intensity 174 increase $(5-8 \%)$ and a $2 \mathrm{~nm}$ blue-shift are observed, whereas 175 upon further addition of $\mathrm{Ag}^{+}$ions the emission intensity 176 decreases by about $10-15 \%$.

Overall, the observed behavior can be ascribed to an initial 178 decoupling of the two porphyrins which move apart from their 179 initial position after the complexation of the $\mathrm{Ag}^{+}$ions and to a 180 subsequent process of formation of a fully complexed 181 $\left[\mathrm{Ag}_{4}(\mathbf{2})\right]^{4+}$ cage, where the two porphyrins are forced to stay 182 in a remote cofacial conformation. The slight decrease in 183 emission intensity is expected on the basis of the "large" 184 distance between the two porphyrins in the fully complexed 185 cage $(0.95 \mathrm{~nm}$, see below). In cofacial porphyrin dimers, in 186 fact, the extent of the decrease scales with the decrease of the 187 interporphyrin distance, and our data are in agreement with a 188 $15 \%$ reduction of emission intensity previously observed for 189 the formation of face-to-face porphyrinic coordination cages 190 where porphyrins are held at a distance of ca. $0.70 \mathrm{~nm}^{26}$ The 191 excited state lifetime measured at the end of titration is $1.7 \mathrm{~ns}, 192$ similar to that of the uncomplexed cage.

193

Titration of cage $1\left(4.9 \times 10^{-7} \mathrm{M}\right)$ with $\mathrm{Ag}^{+}$leads to the 194 absorption changes shown in Figure 3: the Soret band $195 \mathrm{f3}$

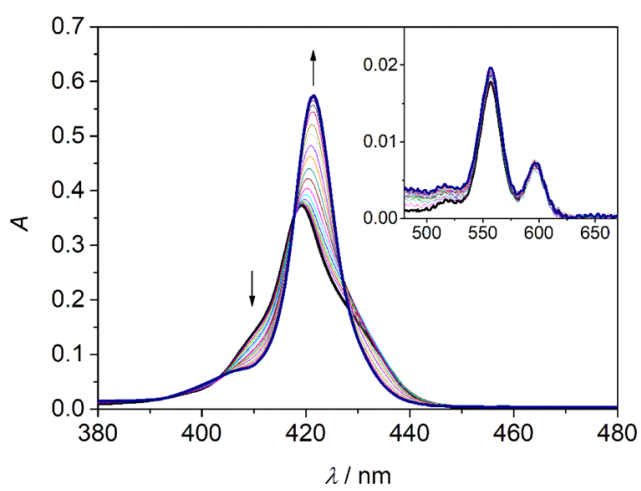

Figure 3. Absorption spectra of a solution of $1\left(4.9 \times 10^{-7} \mathrm{M}\right)$ in $\mathrm{DCM} / \mathrm{MeOH}(90: 10)$ upon addition of $\mathrm{Ag}(\mathrm{OTf}):\left[\mathrm{Ag}^{+}\right]=0-20.2$ equiv (black thick: 0 equiv, blue thick: 20.2 equiv; the arrows indicate the trend of the curves). In the inset, the Q-band region is shown.

sharpens with the formation of a single band with maximum 196 at $421.5 \mathrm{~nm}$. One distinct family of curves with isosbestics at 197 404 and $418 \mathrm{~nm}$ is observed, defining a single equilibrium of 198 complexation. For cage 2, changes in the Q-bands are minimal 199 (Figure 3 inset).

200

Emission spectra, obtained upon excitation at 418 and 556201 $\mathrm{nm}$, show a decrease of intensity on the order of $10 \%$, without 202 spectral shift, when the cage is titrated with $\mathrm{Ag}^{+}$(Figures S5 203 and S6). This outcome resembles that obtained for cage $\mathbf{2}$ and 204 can be ascribed, as well, to the formation of the fully 205 complexed $\left[\operatorname{Ag}_{4}(\mathbf{1})\right]^{4+}$ cage. The extent of the reduction is 206 reasonable for a cofacial porphyrin pair at a distance of 0.85207 $\mathrm{nm}$ (calculated distance, see below). The measured lifetime for 208 the complex is $1.6 \mathrm{~ns}$.

209

By comparing the absorption spectra of the two cages at the 210 end of titration with twice the spectrum of the model $\mathrm{Zn}-211$ alkyne (Figure S7), it can be observed that the spectral shape 212 and the intensity of the Soret band is almost (but not fully) 213 

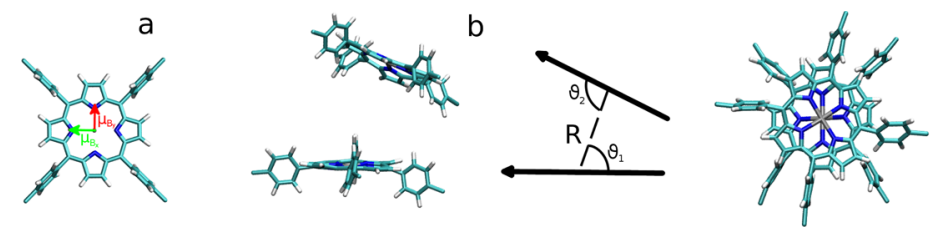

C

Figure 4. (a) Representative structure of Zn-TTP in which the transition dipoles, $\mu_{\mathrm{B}_{x}}$ and $\mu_{\mathrm{B} y}$, are highlighted. Definition of the geometrical parameters defining the reciprocal orientation of the two $\mathrm{Zn}$-porphyrins during the MD simulation of the cages: the distance, $R$, between the two centers of mass, the angles, $\theta_{1}, \theta_{2}$, that is, the angles between each of the Zn-porphyrin planes and the vector connecting the two centers of mass (b), and $\beta$, the angle defining the rotation of the porphyrin planes one with respect to the other (c).

214 recovered in the complexes (the Soret maximum of the 215 complexes is blue-shifted by $4-5 \mathrm{~nm}$ with respect to that of the 216 model). This might confirm that the process of $\mathrm{Ag}^{+}$ 217 complexation separates the two porphyrins in the cages 218 reducing their exciton coupling. Despite the similar features 219 of the spectra of the two complexes, a slight difference in the 220 peak maxima of about $2 \mathrm{~nm}$ is observed. A detailed 221 computational analysis, presented below, clearly describes the 222 origin of the above-mentioned spectral features.

223 In order to derive the association constants for the formation 224 of the silver(I)-complexed cages, the absorption data have 225 been elaborated by means of the ReactLab Equilibria 226 Software. ${ }^{a}$ The emission data could not be treated due to 227 the small spectral changes observed upon titration. The 228 absorption spectral evolution has been treated assuming a 229 single 1:4 (cage $\left./ \mathrm{Ag}^{+}\right)$equilibrium for both cages. With this 230 model, the fitting of the experimental spectra is reasonably 231 good, even for cage 2, where biphasic behavior has been 232 observed; in the latter , the use of models based on 233 consecutive $\left(2+2 \mathrm{Ag}^{+} \leftarrow \overline{\mathrm{T}_{2}}(\mathbf{2})\right]^{2+} ;\left[\mathrm{Ag}_{2}(2)\right]^{2+}+2 \mathrm{Ag}^{+} \leftrightarrow$ $\left.234\left[\mathrm{Ag}_{4}(\mathbf{2})\right]^{4+}\right)$ or parallel $\left(2+2 \mathrm{Ag}^{+} \leftrightarrow\left[\mathrm{Ag}_{2}(\mathbf{2})\right]^{2+} ; \mathbf{2}+4 \mathrm{Ag}^{+} \leftrightarrow\right.$ $\left.235\left[\mathrm{Ag}_{4}(2)\right]^{4+}\right)$ processes did not produce reasonable results. 236 Figure 88 compares the absorption spectrum of cage 2 with 237 the fitted spectrum of $\left[\mathrm{Ag}_{4}(\mathbf{2})\right]^{4+}$ and the end-of-titration 238 spectrum obtained experimentally. The same comparison for 239 cage $\mathbf{1}$ is presented in Figure S9. It can be seen that the fitted 240 spectrum of the complex matches well with the experimental 241 one in both cases. The same good correspondence can be 242 observed by comparing the fitted and experimental spectra at 243 different titration stages: Figures S10 and S11 present this 244 comparison for some representative titration points for cages 2 245 and 1, respectively. The association constants, derived as 246 average of values obtained from the elaboration of different 247 titrations, are $\left[\right.$ in $\left.\log \left(K_{\mathrm{a}} / \mathrm{M}^{-4}\right)\right]: 20.4 \pm 0.5$ and $20.8 \pm 0.9$ for 2482 and 1, respectively.

249 Computational Results. Because of large transition dipole 250 moments in porphyrins, modification of their Soret bands 251 upon their assembly within a system are frequently observed. 252 In symmetric metalloporphyrins, this band results from the 253 superposition of two electronic transitions, $\mathrm{B}_{x}$ and $\mathrm{B}_{y}$, with 254 strong orthogonal transition dipoles in the $\pi$-plane degenerate ${ }_{255}$ in the monomeric state $\left(\mu_{\mathrm{B}_{x}}\right.$ and $\mu_{\mathrm{B} y}$, Figure $\left.4 \mathrm{a}\right)$. When two 256 porphyrins are placed in proximity, then the first set of 257 transition dipoles, $\mu_{\mathrm{B}_{x}}$ and $\mu_{\mathrm{B}_{y}}$, interacts with the second set of ${ }_{258}$ transition dipoles of the other porphyrin, $\mu_{\mathrm{B}_{x}}^{\prime}$ and $\mu_{\mathrm{B}_{y}}^{\prime}$ 259 (hereafter, the prime symbol denotes transition dipole 260 moments belonging to the second molecule). The observed 261 absorption band in the Soret region is the sum of the 262 absorption bands resulting from the excitonic coupling. 263 Therefore, in flexible systems in which the orientation of the assembly is not fixed and has a distribution in space, a broad 264 Soret band is observed due to the presence of various types of 265 interactions among $\mu_{\mathrm{B},}, \mu_{\mathrm{B},}, \mu_{\mathrm{Bx}}^{\prime}$ and $\mu_{\mathrm{By}}^{\prime}$.

266

In what follows, the Soret band of cages $\mathbf{1}$ and $\mathbf{2}$ and their 267 corresponding silver(I)-complexed cages $\left[\mathrm{Ag}_{4}(\mathbf{1})\right]^{4+}$ and 268 $\left[\mathrm{Ag}_{4}(\mathbf{2})\right]^{4+}$, are reconstructed and interpreted with the MD- 269 PMM approach. First, one of the systems (namely, cage 2) is 270 used as the model system to investigate changes in the position 271 and shape of the band as a function of geometrical parameters. 272 Then, the spectra of all systems are reconstructed and 273 interpreted.

Uncomplexed Cages 1 and 2. The Soret band of a dimer of 275 Zn-porphyrins (as in the case of the cages) arises from 276 transitions to four exciton states $\left(S_{2 \mathfrak{a}}, S_{2 b}, S_{2 \mathcal{v}}\right.$ and $\left.S_{2 \mathrm{~d}}\right)$. The 277 position and shape of the Soret band, thus, depend on the 278 excitonic coupling between the two Zn-porphyrins (i.e., on the 279 relative intensity of the single exciton states and the magnitude 280 of their splittings) and are, thus, highly sensitive to differences 281 in conformational states. MD simulations and subsequent 282 calculation of the Soret band of cages $\mathbf{1}$ and 2, thus, allow the 283 characterization of the most probable conformational states for 284 the two cages.

MD simulations of cages $\mathbf{1}$ and $\mathbf{2}$ were performed in DCM/ 286 $\mathrm{MeOH}(90: 10)$ at $300 \mathrm{~K}$ (1 cage molecule per simulation box, 287 that is, infinite dilution) to reproduce the experimental 288 conditions. From the MD simulations, we identified different 289 conformational states on the basis of the geometrical 290 parameters defining the excitonic coupling, that is, the distance 291 $R$ between the two centers of mass of the two Zn-porphyrins 292 and the relative orientation of the transition dipole moments 293 (Figure 4a), the latter defined by the following angles: $\theta_{1}, \theta_{2}, 294$ the angles between each of the $\mathrm{Zn}$-porphyrin plane and the 295 vector connecting the two centers of mass (Figure 4b), and $\beta, 296$ the angle defining the rotation of the porphyrin planes, one 297 with respect to the other (Figure $4 \mathrm{c}$ ).

In order to choose a reduced number of variables to define 299 the conformational states, we analyzed the dependence of the 300 Soret band on the geometrical parameters mentioned above. In 301 the case of the present cages, $\beta$ does not vary much and has a 302 negligible effect on the Soret band shape and position (Figure 303 S12). The bidimensional distribution of $\theta_{1}$ and $\theta_{2}$ calculated 304 from the MD simulation (see Figure 5) shows a strong linear 305 f5 correlation, indicating that the porphyrin planes tend to be 306 parallel to each other, in particular in the region $\theta_{1}, \theta_{2}>40^{\circ}$, in 307 which the planes are more "cofacial". For $\theta_{1}, \theta_{2}<40^{\circ}$ (i.e., for 308 "slipped" conformations), the correlation is lower, indicating 309 the presence of nonparallel (i.e., "oblique") conformations 310 among the slipped conformations. We tested the dependence 311 of the Soret band as a function of different $\theta_{1}$ and $\theta_{2}$ values, 312 both along the $\theta_{1}=\theta_{2}$ diagonal (black) and along the line of 313 equation, $\theta_{1}=65-\theta_{2}$ (red), approximating the most 314 


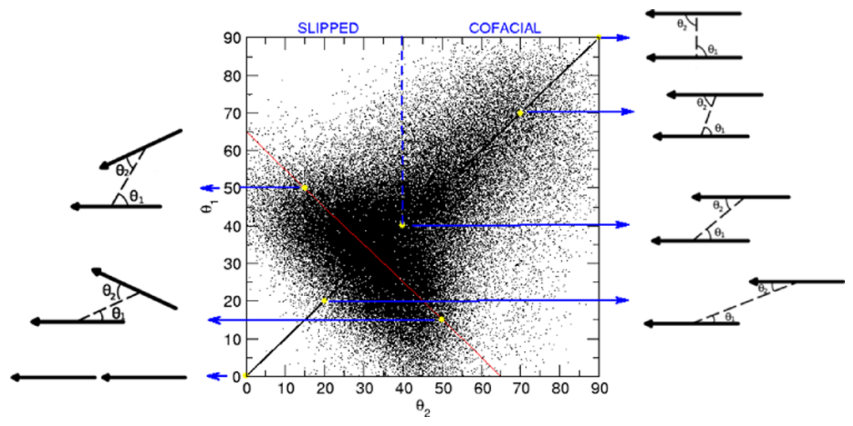

Figure 5. Bidimensional distribution of $\theta_{1}$ and $\theta_{2}$ as calculated from the MD simulation of cage 2. A schematic representation of the reciprocal orientation of the two Zn-porphyrins along the black and red diagonal is also reported.

315 populated basin of the bidimensional distribution of the two 316 angles for $\theta_{1}$ and $\theta_{2}<40^{\circ}$ (Figure 5). Although major changes 317 in the Soret band are observed along the black diagonal, that is, 318 with parallel planes, (see below) only very minor changes in 319 the Soret band are observed along the red diagonal, that is, 320 with an "oblique" orientation of the planes (Figure S13). 321 Finally, as expected, a strong dependence of the Soret band on $322 R$ was also found (see below).

323 On the basis of the results mentioned previously, we used $R$ 324 and $\theta_{1}=\theta_{2}=\theta$ as principal configurational coordinates. In 325 particular, we divided the $\theta_{1}, \theta_{2}$ plane into two regions along 326 the black diagonal (cofacial region, C, $\theta>40^{\circ}$; slipped region, $327 \mathrm{~S}, \theta<40^{\circ}$ ) and for each subpopulation, we considered three 328 different ranges of $R(0.6<R<0.85 \mathrm{~nm}, 0.85<R<1.05 \mathrm{~nm}$ 329 and $R>1.05 \mathrm{~nm})$. An additional state is a "closed" 330 conformation in which $R<0.6 \mathrm{~nm}$ and $\theta$ is "restricted" in 331 the range $\theta<40^{\circ}$. Seven states are thus defined, reported in 332 Table 2.

Table 2. Schematic Representation of the Seven States Defined on the Basis of the Geometrical Parameters $R$ and $\theta$

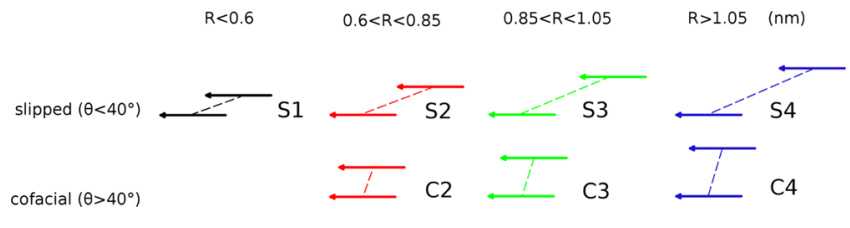

f6 333 In Figure 6 are reported the Soret bands calculated on the 334 subpopulations corresponding to the seven states. In addition, 335 the Soret bands of these states were also calculated, excluding 336 the excitonic coupling in order to have a reference spectrum of 337 a monomeric Zn-porphyrin (similar to the Zn-alkyne model 338 used in the experiments) in which only the effect of the 339 perturbation (i.e., the effect of the rest of the cage and the 340 solvent) is retained. The spectrum of the monomeric reference 341 was almost invariant in the different conformations (peak 342 maximum at $424 \mathrm{~nm}$ and $\varepsilon=5.2 \times 10^{5} \mathrm{M}^{-1} \mathrm{~cm}^{-1}$ ), thus 343 showing only a moderate effect of the perturbation on the 344 spectral features of the Soret band. The spectra reported in 345 Figure 6 are obtained from the simulation of cage 2. A similar 346 analysis was performed for cage $\mathbf{1}$ showing an analogous 347 dependence on $R$ and $\theta$.

348 The "closed" state (S1) shows very peculiar features: the 349 band is blue-shifted (with respect to the peak position of the 350 monomeric reference at $424 \mathrm{~nm}$ ) and the peak is split.
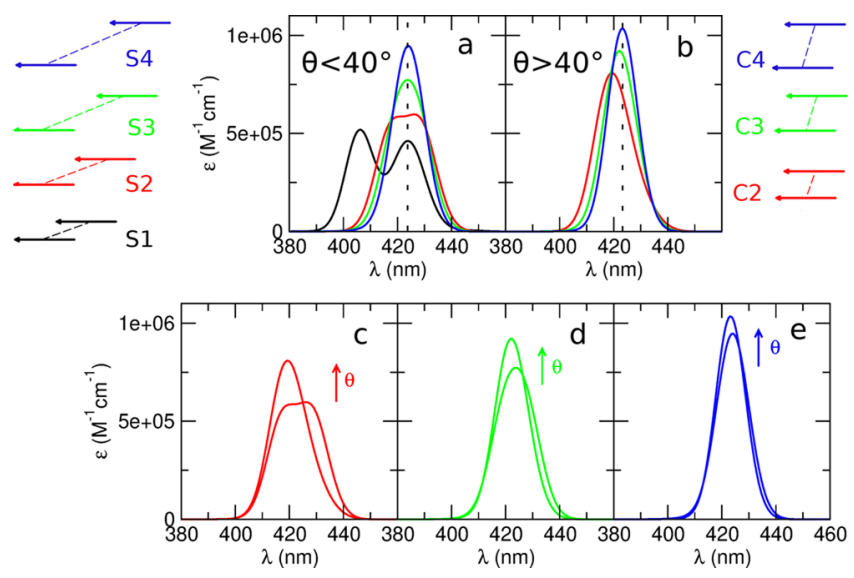

Figure 6. Soret bands calculated on the seven different conformational states identified along the MD simulation of cage 2 and reported in Table 2. (a) Soret bands of states S1, S2, S3, and S4. (b) Soret bands of states C2, C3, and C4. (c) Soret bands of states S2 $(\theta$ $\left.<40^{\circ}\right)$ and $\mathrm{C} 2\left(\theta>40^{\circ}\right)(\mathrm{d})$. Soret bands of states $\mathrm{S} 3\left(\theta<40^{\circ}\right)$ and C3 $\left(\theta>40^{\circ}\right)$. (e) Soret bands of states S4 $\left(\theta<40^{\circ}\right)$ and C4 $(\theta>$ $\left.40^{\circ}\right)$. The dashed line shows the position at $424 \mathrm{~nm}$ of the band for the monomeric reference.

Concerning the other conformations, the following can be 351 observed: (i) for $0.6 \mathrm{~nm}<R<0.85 \mathrm{~nm}$ (red curves) and $\theta<352$ $40^{\circ}$ (slipped conformations), the band has the lowest epsilon 353 and the largest bandwidth (with the peak maximum still 354 showing a slight splitting). (ii) If $\theta$ is kept constant, a relative 355 redshift and an increase of intensity (and a resulting 356 narrowing) is observed for increasing $R$ values, the maximum 357 shift being 4-5 nm (see Figure 6a,b); for $R$ values larger than 358 $1.05 \mathrm{~nm}$, the spectrum of the monomeric reference is basically 359 recovered (peak maximum at $424 \mathrm{~nm}$ and $\varepsilon=2 \times 5.2 \times 10^{5} 360$ $\mathrm{M}^{-1} \mathrm{~cm}^{-1}$ ). (iii) If $R$ is kept constant, a relative red-shift and a 361 decrease of intensity (and a resulting broadening) is observed 362 for decreasing $\theta$ values, the maximum shift being $7 \mathrm{~nm}$ at the 363 lower $R$ (see Figure $6 \mathrm{c}-\mathrm{e}$ ).

We used the spectra of the different states to reconstruct the 365 experimental Soret bands of cages $\mathbf{1}$ and 2. Results are shown 366 in Figure 7. The main results are the following: (i) the spectral $367 \mathrm{f7}$ features of cage $\mathbf{2}$ are mainly due to the presence of slipped 368 conformations $\left(\theta<40^{\circ}\right)$ with $0.6<R<0.85 \mathrm{~nm} 369$ (conformation S2 in Table 2); it is worth noting that the X- 370 ray structure of the crystal of cage 2 belongs to the S2 state 371 (the $\mathrm{Zn}-\mathrm{Zn}$ distance is $0.7 \mathrm{~nm}$ and the two $\mathrm{Zn}$-porphyrins are 372 slipped $^{32}$ ); (ii) the spectral features of cage $\mathbf{1}$ are mainly due to 373 the presence of more cofacial conformations $\left(\theta>40^{\circ}\right), 374$ whereas the $\mathrm{Zn}-\mathrm{Zn}$ distance is similar to the case of cage $\mathbf{2} 375$ (state C2 in Table 2). The longer linkers of cage $\mathbf{2}$ ensure more 376 flexibility to this cage, leading to closer porphyrin planes (mean 377 interplanar distance of $0.4 \mathrm{~nm}$ ) and stronger exciton coupling 378 than in cage $\mathbf{1}$ with shorter linkers (mean interplanar distance 379 of $0.7 \mathrm{~nm}$ ).

Cages 1 and 2 Complexed with $\mathrm{Ag}^{+}$lons. In the fully 381 complexed $\left[\operatorname{Ag}_{4}(\mathbf{1})\right]^{4+}$ and $\left[\operatorname{Ag}_{4}(\mathbf{2})\right]^{4+}$ cages, one $\mathrm{Ag}^{+}$ion is 382 coordinated to each $\varepsilon+1$ e four linkers. The coordination of 383 $\mathrm{Ag}^{+}$ions the link was modeled by performing quantum 384 mechanical (QM) calculations on model systems made of a 385 subpart of the system (i.e., one linker) and one $\mathrm{Ag}^{+}$ion (see 386 Methods section). The geometrical parameters of the most 387 stable, optimized structures of the silver(I)-complexed linkers 388 are reported in the Supporting Information (Figure S14). 389 


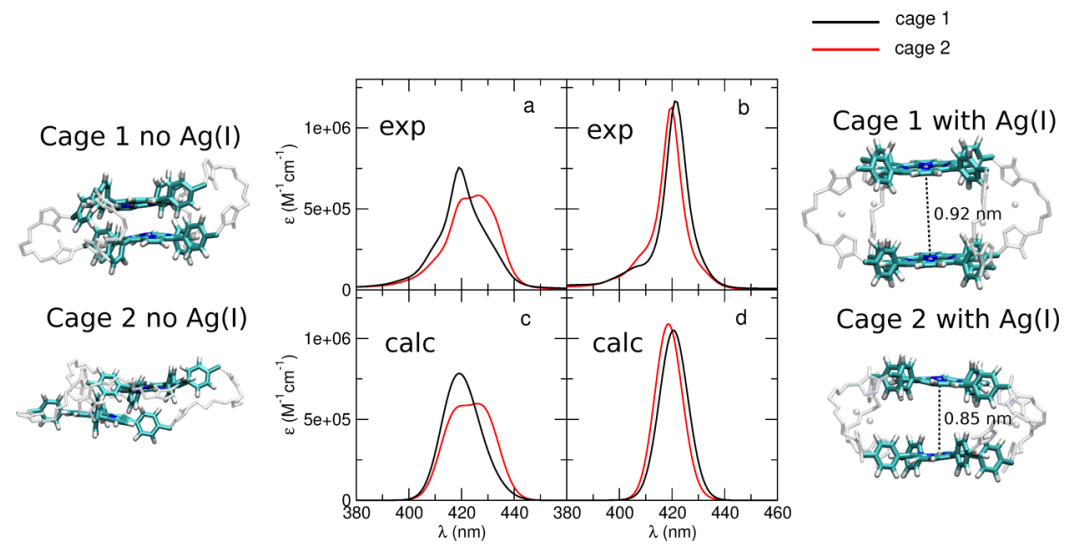

Figure 7. Experimental (a,b) and calculated (c,d) Soret bands for cage $\mathbf{1}$ (black) and cage $\mathbf{2}$ (red) in the absence (a,c) and in the presence (b,d) of $\mathrm{Ag}^{+}$ions coordinated to each of the four linkers. The black curve in panel (c) is the Soret band of conformation S2 of cage 1. The red curve in panel (c) is the Soret band of conformation C2 of cage 2. The Soret bands in panel (d) are calculated using the whole configurational space sampled by the corresponding MD simulations. The dashed line shows the position at $424 \mathrm{~nm}$ of the Soret band for the monomeric reference. Representative snapshots, extracted from the MD simulations of the four systems, of the conformational states that give rise to the calculated spectra are reported.

390 The spectra calculated with the MD-PMM procedure on the $391 \mathrm{MD}$ simulations of $\left[\mathrm{Ag}_{4}(\mathbf{1})\right]^{4+}$ and $\left[\mathrm{Ag}_{4}(\mathbf{2})\right]^{4+}$ cages in DCM/ $392 \mathrm{MeOH}(90: 10)$ are reported in Figure $7 \mathrm{~b}, \mathrm{~d}$. In agreement with 393 the experimental results, the spectra of the complexes are blue394 shifted by 3-4 $\mathrm{nm}$ with respect to the peak maximum of the 395 spectrum of the monomeric reference (i.e., calculated 396 excluding the excitonic coupling) and are narrower than the 397 spectra of the noncomplexed cages. These spectral features are 398 consistent with geometries in which the porphyrins are stacked 399 (cofacial) and at higher distances than in the noncomplexed 400 cages.

401 From the molecular structures of the MD trajectories, it is 402 possible to explain the slight shift between the peak maxima of 403 the two cages: it is related to the distance between the two 404 porphyrins, the mean value of the $\mathrm{Zn}-\mathrm{Zn}$ distance being 405 slightly higher in the complexed cage, $\left[\operatorname{Ag}_{4}(1)\right]^{4+}(0.92 \mathrm{~nm})$ 406 than in the complexed cage, $\left[\operatorname{Ag}_{4}(2)\right]^{4+}(0.85 \mathrm{~nm})$. This 407 difference is likely due to the difference in the coordination of 408 the $\mathrm{Ag}^{+}$ion determined by the presence of the additional $409 \mathrm{CH}_{2}-\mathrm{CH}_{2}-\mathrm{O}$ group in the linker of cage 2, which makes it 410 more flexible with respect to cage 1 allowing the central oxygen 411 atom to enter the coordination sphere of the silver(I) ion. This 412 gives rise to a trigonal geometry in which the distance between 413 the two coordinating nitrogens of the linkers, and the 414 corresponding $\mathrm{N}-\mathrm{Ag}-\mathrm{N}$ angle, is lower in cage 2 than in 415 cage 1 , in which, instead, the $\mathrm{N}-\mathrm{Ag}-\mathrm{N}$ coordination motif is 416 more linear (see Figure S14). This difference in the 417 coordination geometry leads to a slightly smaller distance 418 between the two triazoles (the distance between the two 419 terminal carbon atoms of each linker being $0.99 \mathrm{~nm}$ in cage 2 420 and $1.02 \mathrm{~nm}$ in cage $\mathbf{1}$ ), and, consequently, between the two 421 porphyrins (Figure S14). It must be noticed that the $\mathrm{Zn}-\mathrm{Zn}$ 422 distance found in the MD simulation of $\left[\operatorname{Ag}_{4}(1)\right]^{4+}$ cage gave a $423 \mathrm{Zn}-\mathrm{Zn}$ distance $(0.92 \mathrm{~nm})$ in very good adequacy with the 424 one obtained recently from the X-ray crystallographic structure 425 of this complex $(9.5 \AA) .{ }^{37}$

\section{$426 \square$ METHODS}

427 Absorption and Emission Spectroscopy. Spectroscopic 428 grade $\mathrm{DCM}$ and $\mathrm{MeOH}$ were from Merck and used as 429 received. Silver trifluoromethanesulfonate $(\mathrm{Ag}(\mathrm{OTf}))$, from 430 Sigma-Aldrich, has been stored under argon in a sealed vial in dark and dry conditions. $\mathrm{Ag}(\mathrm{OTf})$ solutions were used fresh 431 and kept in the dark during the measurements. Solutions of 432 cage 2 were filtered with $0.2 \mu \mathrm{m}$ membrane filters (Minisart 433 RC4 filters) before each measurement to avoid aggregation 434 issues. Integrated absorption coefficients were calculated by 435 plotting molar absorption coefficients as a function of 436 absorption energy (in wavenumbers) and calculating the area 437 under the curves.

438

Absorption spectra were recorded with a PerkinElmer 439 Lambda 650 spectrophotometer. Room temperature emission 440 spectra were collected with an Edinburgh FLS920 fluorimeter 441 equipped with a Peltier-cooled Hamamatsu R928 PMT (200- 442 $850 \mathrm{~nm}$ ). Fluorescence quantum yields were evaluated from 443 the area of the luminescence spectra, corrected for the 444 photomultiplier response, with reference to TPP (tetraphe- 445 nylporphyrin) in aerated toluene $\left(\phi_{\mathrm{fl}}=0.11\right) .{ }^{38}$ Measurements 446 at $77 \mathrm{~K}$, performed with the same fluorimeter, made use of 447 Pyrex tubes dipped in liquid nitrogen in a quartz Dewar. Gated 448 emission spectra were acquired by using a time-gated spectral 449 scanning mode and a $\mu \mathrm{F} 920 \mathrm{H}$ Xenon flash lamp (pulse width 450 $<2 \mu$ s, repetition rate between 0.1 and $100 \mathrm{~Hz}$ ) as the 451 excitation source. Spectra were corrected for the wavelength 452 dependent photomultiplier response. Triplet excited state 453 lifetimes were measured with the same apparatus in multi- 454 channel scaling mode.

Titration experiments were performed by incremental 456 addition of micro aliquots of stock solutions of $\mathrm{Ag}$ (OTf) 457 $\left(10^{-3}\right.$ to $\left.10^{-4} \mathrm{M}\right)$ to a solution of molecular cage $\left(5-6 \times 10^{-7} 458\right.$ $\mathrm{M})$. The final volume added was kept below $10 \%$ of the total 459 volume to avoid dilution of the porphyrin. The experiments 460 have been conducted avoiding light exposure of the solutions. 461

Fluorescence lifetimes were measured by means of an IBH 462 time correlated single photon counting apparatus with 463 excitation at $560 \mathrm{~nm}$. Analysis of the decay profiles against 464 time was performed using the Decay Analysis Software DAS6 465 provided by the manufacturer.

466

Estimated errors are $10 \%$ on lifetimes, $10 \%$ on quantum 467 yields, $20 \%$ on molar absorption coefficients, and $3 \mathrm{~nm}$ on 468 emission and absorption peaks.

469

PMM Calculations. The MD-PMM approach is a hybrid 470 quantum/classical theoretical-computational approach, similar 471 in spirit to other hybrid methods, ${ }^{39-41}$ based on MD 472 
473 simulations and on the PMM. In the MD-PMM approach, ${ }^{34,42}$ 474 the part of the system where the quantum processes of interest 475 occur, that is the quantum center (QC), is treated at the 476 electronic level, and the rest of the system is modeled as an 477 atomic-molecular classical subsystem exerting an electrostatic 478 effect on the QC electronic states. The main difference with 479 other hybrid methods is that in the MD-PMM, the whole 480 system (including the QC) phase space is sampled by classical $481 \mathrm{MD}$ simulations based on typical atomistic empirical/semi482 empirical force-fields, allowing an extensive sampling of the 483 QC and environment configurational space. The electrostatic 484 perturbation of the environment is included a posteriori: the 485 electronic properties of the isolated QC (unperturbed 486 properties) are calculated quantum-chemically in vacuum 487 (i.e., in the gas phase) and then, for each configuration 488 generated by all-atoms classical MD simulations of the whole 489 system, the electrostatic effect of the instantaneous atomistic 490 configurations of the environment is included as a perturbing 491 term within the QC Hamiltonian operator. The electronic 492 Hamiltonian operator $\hat{H}$ of the $\mathrm{QC}$ embedded in the 493 perturbing environment can be thus expressed via

$494 \hat{H}=\hat{H}^{0}+\hat{V}$

495 where $\hat{H}^{0}$ is the QC unperturbed electronic Hamiltonian (i.e., 496 as-obtained considering the isolated QC) and $\hat{V}$ is the 497 perturbation operator. In typical PMM calculations, the 498 perturbing electric field provided by the environment atomic 499 charges is used to obtain the perturbation operator, $\hat{V}$ via a 500 multipolar expansion centered in the QC center of mass, $r_{0}$ 501 (QC-based expansion ${ }^{43}$ )

502

$$
\hat{V} \simeq \sum_{j}\left[V\left(r_{0}\right)-E\left(r_{0}\right) \cdot\left(r_{j}-r_{0}\right)\right] q_{j}
$$

503 with $j$ running over all QC particles (i.e., nuclei and electrons), $504 q_{j}$ as the charge of the $j$ th particle, $r_{j}$ as the corresponding 505 coordinates, $V$ as the electrostatic potential exerted by the 506 perturbing environment, and $E=\mathrm{Z} \partial V / \partial r$ as the perturbing 507 electric field.

508 In the present work, we use a very recent development of the 509 PMM approach including higher order terms by expanding the 510 perturbation operator at each atom of the QC (atom-based 511 expansion). ${ }^{43}$ Within such an approach, the perturbation 512 operator, $\hat{V}$ is expanded within each $N$ th atomic region around 513 the corresponding atomic center, $R_{N}$ (i.e., the nucleus position 514 of the $N$ th atom of the $\mathrm{QC})$, providing

$$
\hat{V} \simeq \sum_{N} \sum_{j} \Omega_{N}\left(r_{j}\right)\left[V\left(R_{N}\right)-E\left(R_{N}\right) \cdot\left(r_{j}-R_{N}\right)+\ldots\right] q_{j}
$$

515

516 with $j$ running over all QC nuclei and electron, $N$ running over 517 all QC atoms, and each $\Omega_{N}$ as a step function being null 518 outside and unity inside the Nth atomic region. Such an atom519 based expansion is here used only for the Hamiltonian matrix 520 diagonal elements, whereas the other Hamiltonian matrix 521 elements are obtained by using the QC-based perturbation 522 operator expansion within the dipolar approximation (eq 2). 523 At each frame of the MD simulation, the perturbed 524 electronic Hamiltonian matrix is constructed and diagonalized, 525 providing a continuous trajectory of perturbed eigenvalues and 526 eigenvectors to be used for evaluating the QC instantaneous 527 perturbed quantum observable of interest, for example, 528 excitation energies/absorption frequencies.
In case we deal with a set of interacting chromophores, that 529 is, a set of interacting QCs, we must take into account the 530 possible excitation coupling occurring among the QCs. In the 531 present case, the interaction between the electronic excitations 532 localized on each of the two $\mathrm{Zn}$-porphyrin units has to be 533 considered. To this aim, we use an improved implementation, 534 previously applied to the study of the hyperchromic effect in 535 $\mathrm{DNA}^{44}$ in which the perturbed Hamiltonian operator for the 536 two QCs has to be considered

$$
\tilde{H}=E_{0} \tilde{I}+\Delta \tilde{H}
$$

where $E_{0}$ is the electronic ground-state energy, and $\Delta \tilde{H}$ is the 539 excitation matrix whose diagonal elements are given by the 540 single chromophore perturbed excitation energies. The non- 541 diagonal elements of the excitation matrix, describing the 542 chromophores quantum interaction, are obtained by truncating 543 the expansion of the chromophore interaction operator at the 544 dipolar term. Thus, the electronic coupling between the two 545 QCs is treated as a dipole-dipole interaction and the $k, k^{\prime} 546$ interaction operator is given by

$$
\begin{aligned}
\hat{V}_{k, k^{\prime}}= & \frac{q_{T, k} q_{T, k^{\prime}}}{R_{k, k^{\prime}}}+\frac{q_{T, k^{\prime}} \hat{\mu}_{k^{\prime}} \cdot R_{k, k^{\prime}}}{R_{k, k^{\prime}}{ }^{3}}-\frac{q_{T, k^{\prime}} \hat{\mu}_{k} \cdot R_{k, k^{\prime}}}{R_{k, k^{\prime}}{ }^{3}}+\frac{\hat{\mu}_{k} \cdot \hat{\mu}_{k^{\prime}}}{R_{k, k^{\prime}}{ }^{3}} \\
& -3 \frac{\hat{\mu}_{k^{\prime}} \cdot R_{k, k^{\prime}} \hat{\mu}_{k} \cdot R_{k, k^{\prime}}}{R_{k, k^{\prime}}{ }^{5}}
\end{aligned}
$$

where $\hat{\mu}_{k}$ is the $k$ th chromophore dipole operator, and $R_{k, k^{\prime}}$ is 549 the $k^{\prime}$ to $\mathrm{k}$ chromophore displacement vector defined by the 550 corresponding chromophore origins (typically the centers of 551 mass). It has to be noted that the modeling of the 552 chromophore interaction as a dipole-dipole interaction is a 553 reliable approximation only for interchromophoric distances 554 larger than the dimension of the chromophore. In the present 555 case, the smallest interchromophoric distance is $0.55 \mathrm{~nm}, 556$ whereas the size of the chromophore (i.e., the distance 557 between the center of mass and the most external atoms of the 558 chromophore) is on the order of $0.4 \mathrm{~nm}$. Thus, the dipolar 559 approximation is suitable to describe the excitonic coupling 560 between the porphyrins in the cages, allowing interpreting such 561 a coupling in terms of dipole strengths, relative orientation, 562 and distance (see the Computational Results section). 563 Therefore, in the off-diagonal elements providing the 564 chromophore quantum interaction as expressed by eq 5 , the 565 perturbed transition dipoles are involved, as obtained by 566 considering the single chromophore embedded in the field 567 produced by the other one (and all rest of the environment). 568 Finally, by diagonalizing the excitation matrix, we obtain the 569 perturbed excitation energies and eigenstates (exciton states). 570

To calculate the absorption spectrum, once the perturbed 571 frequencies $(\nu)$ and transition dipoles $\left(\mu_{j, i}\right)$ for each exciton 572 state are obtained at each of the $N$ frames of the trajectory, we 573 evaluate the excitation energy distribution using an appropriate 574 number of bins in the frequency space. The excitation energies 575 distribution and the corresponding transition dipoles are then 576 utilized for calculating the molar extinction coefficient, $\varepsilon_{0, i}$ for 577 the ground to the $i$ th excited state transition, providing the 578 absorption spectra by the equation

$$
\varepsilon_{0, i} \simeq \sum_{\nu_{\mathrm{ref}}} \frac{\Gamma_{\mathrm{A}}\left(\nu_{\mathrm{ref}}\right) h \nu}{N} \frac{\mathrm{e}^{-\left(\nu-\nu_{\mathrm{ref}}\right)^{2} / 2 \sigma^{2}}}{\sigma \sqrt{2 \pi}}
$$




$$
\Gamma_{\mathrm{A}}\left(\nu_{\mathrm{ref}}\right)=\frac{\left|\boldsymbol{\mu}_{0, j}\right|_{\nu_{\text {ref }}}^{2}}{6 \varepsilon_{0} c \hbar^{2}}
$$

582 In these last equations, $\nu_{\text {ref }}, n\left(\nu_{\text {ref }}\right)$, and $\left|\boldsymbol{\mu}_{0, j}\right|_{\nu_{\text {ref }}}{ }^{2}$ are the 583 frequency at the center of each bin, the corresponding number 584 of MD frames and mean transition dipole square norm within 585 the bin. Moreover, $\hbar=h /(2 \pi)$, where $h$ is the Planck constant, $586 \epsilon_{0}$ is the vacuum dielectric constant, $c$ is the light speed, and $\sigma^{2}$ 587 is the variance produced by the semiclassical vibrations 588 neglected in the evaluation of the unperturbed properties. In 589 the present case, the value of $\sigma^{2}$ has been estimated on the 590 basis of the full width at half maximum of the experimental 591 spectrum in toluene, ${ }^{45}$ approximating the vacuum condition. 592 Equations 6 and 7 provide the calculated spectral line shape: 593 the width of the calculated bands (inhomogeneous broad594 ening) is, thus, due to the complete effect of the semiclassical 595 fluctuations of the $\mathrm{QC}$ and its environment.

596 Quantum Mechanical Calculations. To calculate the 597 absorption spectra of cages $\mathbf{1}$ and 2 , and their corresponding 598 silver(I)-complexed cages, $\left[\operatorname{Ag}_{4}(\mathbf{1})\right]^{4+}$ and $\left[\operatorname{Ag}_{4}(2)\right]^{4+}$, the two $599 \mathrm{Zn}$-porphyrins units were selected as QCs. PMM calculations 600 were performed by considering each porphyrin unit embedded 601 in the field produced by the other porphyrin unit, the four 602 flexible spacers, the $\mathrm{Ag}^{+}$ions (when present), and the solvent. 603 QM calculations were performed on zinc(II) tetratolylpor604 phyrins (Zn-TTPs) (with four para-methyl groups on the 605 meso-phenyl of the TPP) in vacuo in order to obtain the 606 unperturbed electronic eigen functions to be used in the PMM 607 calculations. A geometry optimization of $\mathrm{Zn}$-TTP was 608 performed in vacuo at the density functional theory (DFT) 609 level $^{46}$ with the Coulomb-attenuated hybrid exchange610 correlation functional. ${ }^{47}$ The atomic basis sets were as follows: 611 (i) for the zinc atom the LANL2DZ effective core potential for 612 the inner electrons and a double Gaussian basis set of $613(5 \mathrm{~S}, 5 \mathrm{P}, 5 \mathrm{D}) /[3 \mathrm{~S}, 3 \mathrm{P}, 2 \mathrm{D}]$ quality for the valence electrons were 614 used; $^{48}$ (ii) for the hydrogen, carbon, and nitrogen atoms, a 615 standard 6-31+G(d) Gaussian basis set was used. ${ }^{49}$

616 Time-dependent DFT (TD-DFT) was used with the same 617 functional and basis set for evaluating the properties of the 618 involved excited states. The calculations provided the energies, 619 the atomic charges, and dipole moments of the unperturbed 620 electronic ground state and of the first five unperturbed 621 electronic excited states, besides all ground to excited 622 transition dipole moments necessary for the MD-PMM 623 procedure to be applied. Charges are obtained by fitting the 624 classical electrostatic potential outside the molecule to the 625 corresponding QM potential using the electrostatic potential fit 626 procedure (ESP charges). ${ }^{50}$ All QM calculations are carried 627 out using the Gaussian09 package. ${ }^{51}$

628 To achieve a more meaningful comparison between the 629 experimental and MD-PMM calculated absorption spectra, 630 avoiding disagreements and/or deviations due to inaccuracies 631 of the quantum chemical calculations, the QM calculated 632 magnitude of the transition dipoles corresponding to the Soret 633 band excitations was rescaled on the basis of the experimental 634 values in toluene. ${ }^{45}$ In addition, the QM ground state energy 635 was shifted to reproduce the experimental Soret band 636 excitation energies in toluene. In Table S2 of the Supporting 637 Information, the Soret band excitation energies and transition 638 dipole moments of $\mathrm{Zn}$-TPP, both as obtained from the TD639 DFT calculations and after the rescaling based on the 640 experimental data in toluene, are reported.
The same level of theory was used for the geometry 641 optimization and atomic charges calculations performed on the 642 silver(I)-complexed linkers (see below).

643

MD Simulations. MD simulations of cages $\mathbf{1}$ and $\mathbf{2}$ and 644 their corresponding silver(I)-complexed cages, $\left[\operatorname{Ag}_{4}(\mathbf{1})\right]^{4+}$ and 645 $\left[\mathrm{Ag}_{4}(2)\right]^{4+}$, were performed in $\mathrm{DCM} / \mathrm{MeOH}(90: 10)$ to 646 mimic the experimental conditions. The initial structure of the 647 uncomplexed cage $\mathbf{2}$ was taken from the structure of the X-ray 648 crystal. $^{32}$ To generate the initial structure for the silver(I)- 649 complexed cage, one of the most open conformations 650 generated along the MD simulation of the uncomplexed cage 651 was taken and the four $\mathrm{Ag}^{+}$ions were placed between the two 652 coordinating nitrogen atoms of the linkers; then, this structure 653 was energy minimized using the steepest descent algorithm. 654 The initial structures for both complexed and uncomplexed 655 forms of cage 1 were taken from the crystal structure of the 656 complexed cage (for the uncomplexed simulations, the four 657 $\mathrm{Ag}^{+}$ions were removed). ${ }^{37}$

658

MD simulations were performed with the GROMACS 659 software package using the GROMOS96 [36] force field. The 660 GROMOS force field parameters for uncomplexed cages $\mathbf{1}$ and 661 2 were obtained from the Automated Topology Builder (ATB) 662 database, $^{52}$ with the exception of the partial charges set, 663 obtained from QM calculations (mentioned earlier). For cages 664 $\mathbf{1}$ and $\mathbf{2}$ in the presence of $\mathrm{Ag}^{+}$ions coordinated to each of the 665 four linkers, the force field parameters for the silver(I)- 666 complexed linkers have been obtained as follows. For cage 2, a 667 QM geometry optimization was performed on a model 668 structure of the linker in the presence of one $\mathrm{Ag}^{+}$ion obtained 669 by the above-mentioned energy minimization (see Figure 670 S14). For cage 1, a QM geometry optimization was performed 671 on the silver(I)-complexed linker structure taken from the X- 672 ray crystal (Figure S14). For both cages, the bond lengths and 673 angles, provided by the optimized configuration, were used as 674 geometrical parameters for the subsequent $\mathrm{MD}$ simulations. 675 QM-derived ESP charges for the silver(I)-complexed linkers 676 were used. The remaining force field parameters were obtained 677 by analogy with similar already parametrized chemical groups. 678

The four cages were placed in a dodecahedral box large 679 enough to contain the molecule and at least $1.0 \mathrm{~nm}$ of solvent 680 on all sides with a proper number of DCM molecules to 681 reproduce the density of DCM at $300 \mathrm{~K}$ and $1 \mathrm{bar}(1.33 \mathrm{~g} / 682$ $\mathrm{cm}^{3}$ ). A proper number of DCM molecules was then 683 substituted with the corresponding number of $\mathrm{MeOH} 684$ molecules to reproduce the experimental $\mathrm{DCM} / \mathrm{MeOH} 685$ (90:10) proportion. The force field parameters for DCM and 686 $\mathrm{MeOH}$ were taken from the GROMACS topology database. 687 Simulations were carried out in the NVT ensemble at a 688 constant temperature of $300 \mathrm{~K}$ using the velocity rescaling 689 temperature coupling. ${ }^{53}$ The LINCS algorithm ${ }^{54}$ was used to 690 constrain bond lengths and a time step of 2 fs for numerical 691 integration of the equations of motion was used. The particle 692 mesh Ewald method ${ }^{55}$ was used for the calculation of the long- 693 range interactions and a cut-off of $1.1 \mathrm{~nm}$ was used. After a 694 solute optimization and a subsequent solvent relaxation, each 695 system was gradually heated from 50 to $300 \mathrm{~K}$ using short MD 696 simulations. The trajectories were then propagated for $100 \mathrm{~ns} 697$ for each system. Coordinates are saved at every 1 ps.

698

\section{CONCLUSIONS}

699

A combined spectroscopic/computational study of two 700 covalent cages consisting of two zinc(II)-porphyrins connected 701 by four flexible linkers, that have different lengths in the two 702 
703 systems, and the conformational changes induced by binding 704 of four silver(I) ions to the peripheral ligands is reported. The 705 complexation processes are characterized in detail by means of 706 absorption and emission spectroscopy in diluted solutions. The 707 computational MD-PMM procedure used here to interpret the 708 spectroscopic absorption data allows to quantitatively 709 reproduce the essential features of the experimental Soret 710 bands and to characterize the molecular interactions 711 responsible for the changes in the UV-visible absorption.

712 The Soret band of cage $\mathbf{2}$ with longer linkers results to be 713 red-shifted by approximately $7-8 \mathrm{~nm}$, broader, and slightly 714 split, with respect to that of cage 1 with shorter linkers. The 715 MD-PMM calculations show that these differences are due to 716 the presence of more slipped porphyrinic planes in the cage 717 with longer linkers, whereas the $\mathrm{Zn}-\mathrm{Zn}$ distance is similar in 718 both cages (in the range of $0.6-0.85 \mathrm{~nm}$ ). Upon complexation 719 with the silver(I) ions, it can be observed that the spectral 720 shape and the intensity of the Soret band of both cages almost 721 converge to those of the monomeric reference (in which no 722 excitonic coupling is possible) due to a reduction in the 723 excitonic coupling. This is due to binding of the silver(I) ions, 724 which opens the flattened structures and locks the two 725 porphyrins in a face-to-face disposition. Although the spectral 726 features of both complexed cages are similar, a slight difference 727 in the peak maxima of about $2 \mathrm{~nm}$ is observed and can be 728 attributed to a slightly higher $\mathrm{Zn}-\mathrm{Zn}$ distance in the cage with 729 shorter linkers. It is noteworthy that these structural features 730 are consistent with the reported X-ray crystallographic 731 structures of cage $2^{32}$ and silver(I)-complexed cage $732\left[\operatorname{Ag}_{4}(\mathbf{1})\right]^{4+} \cdot 37$

\section{ASSOCIATED CONTENT}

\section{S Supporting Information}

735 The Supporting Information is available free of charge on the 736 ACS Publications website at DOI: 10.1021/acs.jpcc.9b00742.

737 Absorption, emission, and luminescence data and figures 738 related to computational studies (MD and QM) (PDF)

\section{$739 \square$ AUTHOR INFORMATION}

\section{Corresponding Authors}

741 *E-mail: v.heitz@unistra.fr (V.H.).

742 *E-mail: barbara.ventura@isof.cnr.it (B.V.).

743 *E-mail: isabella.daidone@univaq.it (I.D.).

744 ORCID $\odot$

745 Laura Zanetti-Polzi: 0000-0002-2550-4796

746 Stéphanie Durot: 0000-0001-8815-0422

747 Valérie Heitz: 0000-0002-5828-9199

748 Barbara Ventura: 0000-0002-8207-1659

749 Isabella Daidone: 0000-0001-8970-8408

\section{Author Contributions}

751 The manuscript was written through contributions of all 752 authors. All authors have given approval to the final version of 753 the manuscript.

754 Notes

755 The authors declare no competing financial interest.

\section{ACKNOWLEDGMENTS}

757 The International Center for Frontier Research in Chemistry, 758 icFRC (www.icfrc.fr), and the LabEx-CSC are gratefully 759 acknowledged for funding the Ph.D positions of L.S. The 760 Ministry of Education and Research is acknowledged for a
Ph.D. grant to R.D. We also thank the ANR Agency for the 761 funding of the project ANR 14-CE06-0010 "Switchable cages", 762 the Italian CNR (Project "PHEEL"), and MIUR-CNR project 763 Nanomax N-CHEM. We thank Prof. Massimiliano Aschi for 764 having provided the code for the excitonic coupling 765 calculations and Dr. Ilse Manet (ISOF-CNR) for helpful 766 discussion.

\section{ABBREVIATIONS}

MD, molecular dynamics; PMM, perturbed matrix method; 769 TPP, tetraphenylporphyrin; DCM, dichloromethane; TTP, 770 tetratolylporphyrins

771

\section{ADDITIONAL NOTE}

772

${ }^{a}$ ReactLab EQUILIBRIA 1.1-Global Analysis and Reaction 773 Modelling for Chemical Equilibria. Jplus Consulting Pty Ltd. 774

\section{REFERENCES}

775

(1) Huber, R. A Structural Basis of Light Energy and Electron 776 Transfer in Biology(Nobel Lecture). Angew. Chem., Int. Ed. Engl. 777 1989, 28, 848-869.

(2) Deisenhofer, J.; Michel, H. The Photosynthetic Reaction Center 779 from the Purple BacteriumRhodopseudomonas Viridis(Nobel Lec- 780 ture). Angew. Chem., Int. Ed. Engl. 1989, 28, 829-847. 781

(3) Deisenhofer, J.; Epp, O.; Sinning, I.; Michel, H. Crystallographic 782 refinement at $2.3 \AA$ Resolution and Refined Model of the 783 Photosynthetic Reaction Centre from Rhodopseudomonas viridis. J. 784 Mol. Biol. 1995, 246, 429-457.

(4) Papiz, M. Z.; Prince, S. M.; Howard, T.; Cogdell, R. J.; Isaacs, N. 786 W. The Structure and Thermal Motion of the B800-850 LH2 787

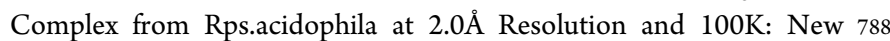
Structural Features and Functionally Relevant Motions. J. Mol. Biol. 789 2003, 326, 1523-1538.

(5) Roszak, A. W. Crystal Structure of the RC-LH1 Core Complex 791 from Rhodopseudomonas Palustris. Science 2003, 302, 1969-1972. 792

(6) Umena, Y.; Kawakami, K.; Shen, J.-R.; Kamiya, N. Crystal 793 structure of oxygen-evolving photosystem II at a resolution of $1.9 \AA .794$ Nature 2011, 473, 55-60.

(7) Pšenčík, J.; Butcher, S. J.; Tuma, R. Chlorosomes: Structure, 796 Function and Assembly. In The Structural Basis of Biological Energy 797 Generation; Hohmann-Marriott, M. F., Ed.; Springer Netherlands: 798 Dordrecht, 2014; Vol. 39, pp 77-109.

(8) Mirkovic, T.; Ostroumov, E. E.; Anna, J. M.; van Grondelle, R.; 800 Govindjee; Scholes, G. D. Light Absorption and Energy Transfer in 801 the Antenna Complexes of Photosynthetic Organisms. Chem. Rev. 802 2017, 117, 249-293.

803

(9) Balaban, T. S. Tailoring Porphyrins and Chlorins for Self- 804 Assembly in Biomimetic Artificial Antenna Systems. Acc. Chem. Res. 805 2005, 38, 612-623.

(10) Li, W.-S.; Kim, K. S.; Jiang, D.-L.; Tanaka, H.; Kawai, T.; 807 Kwon, J. H.; Kim, D.; Aida, T. Construction of Segregated Arrays of 808 Multiple Donor and Acceptor Units Using a Dendritic Scaffold: 809 Remarkable Dendrimer Effects on Photoinduced Charge Separation. 810 J. Am. Chem. Soc. 2006, 128, 10527-10532.

(11) Harvey, P. D.; Stern, C.; Gros, C. P.; Guilard, R. The 812 Photophysics and Photochemistry of Cofacial Free Base and 813 Metallated Bisporphyrins Held Together by Covalent Architectures. 814 Coord. Chem. Rev. 2007, 251, 401-428.

(12) Nakamura, Y.; Aratani, N.; Osuka, A. Cyclic Porphyrin Arrays 816 as Artificial Photosynthetic Antenna: Synthesis and Excitation Energy 817 Transfer. Chem. Soc. Rev. 2007, 36, 831.

(13) Satake, A.; Kobuke, Y. Artificial Photosynthetic Systems: 819 Assemblies of Slipped Cofacial Porphyrins and Phthalocyanines 820 Showing Strong Electronic Coupling. Org. Biomol. Chem. 2007, 5, 821 1679.

(14) Gust, D.; Moore, T. A.; Moore, A. L. Solar Fuels via Artificial 823 Photosynthesis. Acc. Chem. Res. 2009, 42, 1890-1898. 
825 (15) Wasielewski, M. R. Self-Assembly Strategies for Integrating 826 Light Harvesting and Charge Separation in Artificial Photosynthetic 827 Systems. Acc. Chem. Res. 2009, 42, 1910-1921.

828 (16) Lindsey, J. S.; Bocian, D. F. Molecules for Charge-Based 829 Information Storage. Acc. Chem. Res. 2011, 44, 638-650.

830 (17) Pellegrin, Y.; Odobel, F. Molecular Devices Featuring 831 Sequential Photoinduced Charge Separations for the Storage of 832 Multiple Redox Equivalents. Coord. Chem. Rev. 2011, 255, 25788332593.

834 (18) Sprafke, J. K.; Kondratuk, D. V.; Wykes, M.; Thompson, A. L.; 835 Hoffmann, M.; Drevinskas, R.; Chen, W.-H.; Yong, C. K.; Kärnbratt, 836 J.; Bullock, J. E.; et al. Belt-Shaped $\pi$-Systems: Relating Geometry to 837 Electronic Structure in a Six-Porphyrin Nanoring. J. Am. Chem. Soc. 838 2011, 133, 17262-17273.

839 (19) Griffith, M. J.; Sunahara, K.; Wagner, P.; Wagner, K.; Wallace, 840 G. G.; Officer, D. L.; Furube, A.; Katoh, R.; Mori, S.; Mozer, A. J. 841 Porphyrins for Dye-Sensitised Solar Cells: New Insights into 842 Efficiency-Determining Electron Transfer Steps. Chem. Commun. $8432012,48,4145$.

844 (20) Wytko, J. A.; Ruppert, R.; Jeandon, C.; Weiss, J. Metal845 Mediated Linear Self-Assembly of Porphyrins. Chem. Commun. 2018, $84654,1550-1558$.

847 (21) Durot, S.; Taesch, J.; Heitz, V. Multiporphyrinic Cages: 848 Architectures and Functions. Chem. Rev. 2014, 114, 8542-8578.

849 (22) Hong, S.; Rohman, M. R.; Jia, J.; Kim, Y.; Moon, D.; Kim, Y.; 850 Ko, Y. H.; Lee, E.; Kim, K. Porphyrin Boxes: Rationally Designed 851 Porous Organic Cages. Angew. Chem. 2015, 127, 13439-13442.

852 (23) Yu, C.; Long, H.; Jin, Y.; Zhang, W. Synthesis of Cyclic 853 Porphyrin Trimers through Alkyne Metathesis Cyclooligomerization 854 and Their Host-Guest Binding Study. Org. Lett. 2016, 18, 29468552949.

856 (24) Hwang, I.-W.; Kamada, T.; Ahn, T. K.; Ko, D. M.; Nakamura, 857 T.; Tsuda, A.; Osuka, A.; Kim, D. Porphyrin Boxes Constructed by 858 Homochiral Self-Sorting Assembly: Optical Separation, Exciton 859 Coupling, and Efficient Excitation Energy Migration. J. Am. Chem. 860 Soc. 2004, 126, 16187-16198.

861 (25) Hernández-Eguía, L. P.; Escudero-Adán, E. C.; Pintre, I. C.; 862 Ventura, B.; Flamigni, L.; Ballester, P. Supramolecular Inclusion 863 Complexes of Two Cyclic Zinc Bisporphyrins with C60 and C70: 864 Structural, Thermodynamic, and Photophysical Characterization. 865 Chem.-Eur. J. 2011, 17, 14564-14577.

866 (26) Durot, S.; Flamigni, L.; Taesch, J.; Dang, T. T.; Heitz, V.; 867 Ventura, B. Synthesis and Solution Studies of Silver(I)-Assembled 868 Porphyrin Coordination Cages. Chem.-Eur. J. 2014, 20, 9979-9990. 869 (27) Telfer, S. G.; McLean, T. M.; Waterland, M. R. Exciton 870 Coupling in Coordination Compounds. Dalton Trans. 2011, 40, 3097. 871 (28) Yamada, Y.; Nawate, K.; Maeno, T.; Tanaka, K. Intramolecular 872 Strong Electronic Coupling in a Discretely H-Aggregated Phthalo873 cyanine Dimer Connected with a Rigid Linker. Chem. Commun. 2018, 874 54, 8226-8228.

875 (29) Ribó, J. M.; Bofill, J. M.; Crusats, J.; Rubires, R. Point-Dipole 876 Approximation of the Exciton Coupling Model Versus Type of 877 Bonding and of Excitons in Porphyrin Supramolecular Structures. 878 Chem.-Eur. J. 2001, 7, 2733-2737.

879 (30) Hunter, C. A.; Sanders, J. K. M.; Stone, A. J. Exciton Coupling 880 in Porphyrin Dimers. Chem. Phys. 1989, 133, 395-404.

881 (31) Tran-Thi, T. H.; Lipskier, J. F.; Maillard, P.; Momenteau, M.; 882 Lopez-Castillo, J. M.; Jay-Gerin, J. P. Effect of the Exciton Coupling 883 on the Optical and Photophysical Properties of Face-to-Face 884 Porphyrin Dimer and Trimer: A Treatment Including the Solvent 885 Stabilization Effect. J. Phys. Chem. 1992, 96, 1073-1082.

886 (32) Kocher, L.; Durot, S.; Heitz, V. Control of the Cavity Size of 887 Flexible Covalent Cages by Silver Coordination to the Peripheral 888 Binding Sites. Chem. Commun. 2015, 51, 13181-13184.

889 (33) Schoepff, L.; Kocher, L.; Durot, S.; Heitz, V. Chemically 890 Induced Breathing of Flexible Porphyrinic Covalent Cages. J. Org. 891 Chem. 2017, 82, 5845-5851.
(34) Amadei, A.; D’Alessandro, M.; D’Abramo, M.; Aschi, M. 892 Theoretical Characterization of Electronic States in Interacting 893 Chemical Systems. J. Chem. Phys. 2009, 130, 084109.

(35) Zanetti-Polzi, L.; Aschi, M.; Daidone, I.; Amadei, A. Theoretical 895 Modeling of the Absorption Spectrum of Aqueous Riboflavin. Chem. 896 Phys. Lett. 2017, 669, 119-124.

(36) Curutchet, C.; Mennucci, B. Quantum Chemical Studies of 898 Light Harvesting. Chem. Rev. 2017, 117, 294-343.

(37) Djemili, R.; Kocher, L.; Durot, S.; Peuroren, A.; Rissanen, K.; 900 Heitz, V. Positive Allosteric Control of Guests Encapsulation by Metal 901 Binding to Covalent Porphyrin Cages. Chem.-Eur. J. 2019, 25, 1481. 902

(38) Seybold, P. G.; Gouterman, M. Porphyrins. J. Mol. Spectrosc. 903 1969, 31, 1-13.

(39) Gao, J.; Truhlar, D. G. Quantum Mechanical Methods for 905 Enzyme Kinetics. Annu. Rev. Phys. Chem. 2002, 53, 467-505. 906

(40) Vreven, T.; Morokuma, K. Hybrid Methods: ONIOM- 907 (QM:MM) and QM/MM. In Annual Reports in Computational 908 Chemistry; Spellmeyer, D. C., Ed.; Elsevier, 2006; Chapter 3, Vol. 2, 909 pp 35-51. 910

(41) Senn, H. M.; Thiel, W. QM/MM Studies of Enzymes. Curr. 911 Opin. Chem. Biol. 2007, 11, 182-187.

(42) Aschi, M.; Spezia, R.; Di Nola, A.; Amadei, A. A First-Principles 913 Method to Model Perturbed Electronic Wavefunctions: The Effect of 914 an External Homogeneous Electric Field. Chem. Phys. Lett. 2001, 344, 915 374-380.

916

(43) Zanetti-Polzi, L.; Del Galdo, S.; Daidone, I.; D’Abramo, M.; 917 Barone, V.; Aschi, M.; Amadei, A. Extending the Perturbed Matrix 918 Method beyond the Dipolar Approximation: Comparison of Different 919 Levels of Theory. Phys. Chem. Chem. Phys. 2018, 20, 24369-24378. 920

(44) D'Abramo, M.; Castellazzi, C. L.; Orozco, M.; Amadei, A. On 921 the Nature of DNA Hyperchromic Effect. J. Phys. Chem. B 2013, 117, 922 8697-8704.

923

(45) Ventura, B.; Flamigni, L.; Marconi, G.; Lodato, F.; Officer, D. 924 L. Extending the porphyrin core: synthesis and photophysical 925 characterization of porphyrins with $\pi$-conjugated $\beta$-substituents. 926 New J. Chem. 2008, 32, 166-178.

927

(46) Parr, R. G.; Yang, W. Density-Functional Theory of the 928 Electronic Structure of Molecules. Annu. Rev. Phys. Chem. 1995, 46, 929 701-728.

930

(47) Yanai, T.; Tew, D. P.; Handy, N. C. A new hybrid exchange- 931 correlation functional using the Coulomb-attenuating method (CAM- 932 B3LYP). Chem. Phys. Lett. 2004, 393, 51-57.

(48) Hay, P. J.; Wadt, W. R. Ab Initio Effective Core Potentials for 934 Molecular Calculations. Potentials for $\mathrm{K}$ to $\mathrm{Au}$ Including the 935 Outermost Core Orbitals. J. Chem. Phys. 1985, 82, 299-310. 936

(49) Krishnan, R.; Binkley, J. S.; Seeger, R.; Pople, J. A. Self- 937 consistent molecular orbital methods. XX. A basis set for correlated 938 wave functions. J. Chem. Phys. 1980, 72, 650-654.

(50) Besler, B. H.; Merz, K. M.; Kollman, P. A. Atomic Charges 940 Derived from Semiempirical Methods. J. Comput. Chem. 1990, 11, 941 431-439. 942

(51) Frisch, M. J.; Trucks, G. W.; Schlegel, H. B.; Scuseria, G. E.; 943 Robb, M. A.; Cheeseman, J. R. Gaussian 09, Revision E. 01; Gaussian 944 Inc.: Wallingford, CT, USA, 2009.

(52) Malde, A. K.; Zuo, L.; Breeze, M.; Stroet, M.; Poger, D.; Nair, 946 P. C.; Oostenbrink, C.; Mark, A. E. An Automated Force Field 947 Topology Builder (ATB) and Repository: Version 1.0. J. Chem. 948 Theory Comput. 2011, 7, 4026-4037.

(53) Bussi, G.; Donadio, D.; Parrinello, M. Canonical Sampling 950 through Velocity Rescaling. J. Chem. Phys. 2007, 126, 014101.

(54) Hess, B.; Bekker, H.; Berendsen, H. J. C.; Fraaije, J. G. E. M. 952 LINCS: A Linear Constraint Solver for Molecular Simulations. J. 953 Comput. Chem. 1997, 18, 1463-1472.

954

(55) Darden, T.; York, D.; Pedersen, L. Particle Mesh Ewald: An N. 955 $\log (\mathrm{N})$ Method for Ewald Sums in Large Systems. J. Chem. Phys. 956 1993, 98, 10089-10092. 